\title{
MEMORIA Y DIBUJOS INÉDITOS DE UNA LÁMPARA BARROCA DE PLATA QUE PERTENECIÓ AL SAGRARIO DE LA CATEDRAL DE SEVILLA
}

\section{REPORT AND UNPUBLISHED DRAWINGS OF A SILVER BAROQUE LAMP BELONGING TO THE SAGRARIO OF SEVILLE CATHEDRAL.}

\author{
Manuel Varas Rivero \\ Universidad de Sevilla, España \\ mvr@us.es
}

Se da a conocer en este trabajo un documento inédito con dibujos del siglo XVIII relativo a una lámpara barroca de plata que perteneció al Sagrario de la Catedral de Sevilla.

Palabras claves: Documento, inédito, dibujos, lámpara, barroco, platería, s. XVIII.

This work makes public an unpublished document with drawings from the XVIII century related to a silver baroque lamp belonging to the Sagrario of Seville Cathedral.

Key words: Document, unpublished, drawings, lamp, baroque, silversmithing, XVIII century.

El patrimonio suntuario, de gran vulnerabilidad y sometido a frecuentes procesos de fundición que han mermado los tesoros, en muchas ocasiones sólo puede ser conocido a través de documentos y raramente por algún dibujo. En este trabajo, precisamente, analizamos un documento y unos dibujos inéditos que permiten conocer el aspecto de una gran lámpara barroca, hoy desaparecida, y con ello entrever la riqueza suntuaria que alcanzó la Iglesia del Sagrario de la Catedral de Sevilla, un templo especialmente dañado por las pérdidas de patrimonio.

El documento, que aparece denominado como "memoria", documenta todas las piezas de que se componía la lámpara mediante una detallada descripción. Parece inventariar la obra tras un proceso de limpieza (o de restauración, aunque nada se dice que manifieste esa acción). También podría ser parte de una 
catalogación más general, aunque resulta demasiado detallado y se sugiere que la pieza fue descolgada.

No aporta datos el documento respecto al patrocinio, la autoría o la fecha de ejecución de la lámpara. La data del mismo, 1743, el estilo y las operaciones que se intuyen se realizan con la pieza la sitúan en torno al año 1700 o el primer cuarto del s. XVIII.

Pasamos a continuación a analizar el contenido del citado documento, hallado en el Archivo de la Catedral de Sevilla ${ }^{1}$. Consta de seis páginas sin foliar. Junto al texto, en campos reservados, aparecen tres dibujos de las partes principales de la lámpara (cabeza, cadena y vaso principal), que se corresponden con las tres partes en que se ha dividido el propio texto. El modelo de lámpara es el habitual "de plato" que venía dándose en Sevilla desde la Edad Media².

La primera parte describe la cabeza o "manipulo" de la lámpara, es decir el cuerpo que permite colgarla, y ofrece un dibujo o "medio perfil...bosquejado", sistema gráfico de origen arquitectónico que ya era tradicional en el ámbito de la platería desde el s. $\mathrm{XVI}^{3}$. (Figura 1) Esta pieza ofrece un perfil hexagonal en su cuerpo principal que remite al gusto barroco local, aunque su uso será minoritario frente a las formas circulares ${ }^{4}$. El resto de la estructura es la habitual en la época : de sección circular, alterna toros, medias cañas y escocias que, unidos a un cuerpo periforme típicamente barroco, otorgan dinamismo y fluidez a toda la pieza. En los ángulos del cuerpo principal se situaban los enganches para las cadenas, que se proyectaban hacia afuera formando "cartelas" voladas, otro aspecto propio del Barroco.

Importante nota a destacar es el sistema decorativo. Se emplean "florecillas" y hojas de acanto como motivos principales. Las primeras, distribuidas por toda la pieza, son frontales y muestran 4 o 5 pétalos, las segundas, muy abundantes, cubren buena parte del cuerpo principal, el cuerpo superior periforme y aparecen formando los enganches para las cadenas. Sobre los enganches iban cabezas de "serafines" (que no se aprecian bien en el dibujo) ${ }^{5}$. Todos estos elementos

${ }^{1}$ ACS (Archivo de la Catedral de Sevilla), Fondo Histórico General, legajo 11306, 1743, 6 folios sin numerar (en realidad son 3 folios con sus anversos y reversos).

2 SANZ SERRANO, María Jesús: La orfebrería sevillana del Barroco, Sevilla, 1976, t. I, p. 149 .

${ }^{3}$ ACS, Fondo Histórico..., leg. 11306, s. f. Por razones prácticas numeramos los folios. Esta parte ocupa el primer folio y su reverso parcialmente. Este sistema gráfico ya fue incorporado, siguiendo modelos italianos y su propia inventiva, por Juan de Arfe y Villafañe en su obra De Varia Commensuracion..., obra muy reeditada hasta el s. XIX desde su publicación en 1585-87. Véase VARAS RIVERO, Manuel: "Algunas consideraciones sobre la teoría arquitectónica de Juan de Arfe y Villafañe : Clasicismo y método gráfico", $T e$ mas de Estética y Arte, $\mathrm{n}^{\circ}$ XXII, 2008, pp. 93-119.

${ }^{4}$ SANZ SERRANO, M ${ }^{\mathrm{a}}$ Jesús: La orfebrería sevillana..., op. cit., p. 233.

${ }^{5}$ ACS, Fondo Histórico..., leg. 11306, s. f., (primer folio y reverso). 
responden a las modas de la orfebrería sevillana del Barroco pleno, pero su tratamiento técnico es peculiar ${ }^{6}$. La decoración es mayoritariamente sobrepuesta: las abundantes flores van atornilladas, soldadas o bien son propiamente tornillos y los acantos alargados del cuerpo periforme van atornillados, no especificándose nada para el resto de hojas de acanto. Tan solo se habla de cincelado en las pequeñas piezas inferiores del conjunto, sin indicar nada respecto al repujado, técnica que despunta ya en la época (tal vez empleado en los platos afrontados que forman el cuerpo principal, del que no se dice nada respecto a esta cuestión).

La segunda parte describe las cadenas de la lámpara, que unen el cuerpo anterior y el "plato" de luz, en un texto separado y acompañado del dibujo de una de las cadenas $^{7}$ (Figura 2).

Siguiendo los usos del momento, eran piezas muy elaboradas, pues se formaba cada cadena con "diez y ocho piesas redondas de diferentes tamaños y figuras", es decir, diversos boceles, medias cañas y piezas troncocónicas alternados, que además se adornaban con "veinte y sinco florecillas redondas de chapa", unas encajadas en la "vara" central de la cadena y otras sobrepuestas sobre las piezas". Las cadenas tenían un esquema tripartito ("triplicado") que les daba un aspecto abalaustrado : dos tercios en los extremos separados por un tercio central flanqueado por "macollas" recubiertas con largas hojas de acanto sobrepuestas. El tercio central llevaba también flores soldadas de adorno 9 . Finalmente, se situaba en el extremo inferior un cogollo de hojas colgantes que ocultaba el enganche al "plato" de la lámpara.

La decoración, de nuevo centrada en flores frontales de 4 o 5 pétalos y en hojas de acanto, vuelve a aplicarse mediante el sobrepuesto y el atornillado, más acordes en este caso con el carácter calado y articulado de estas piezas ${ }^{10}$.

Los últimos cuatro folios se dedican a describir con detenimiento el vaso principal o "plato" de la lámpara, incorporando texto y dibujo ${ }^{11}$ (Figura 3).

La estructura completa vuelve a mostrar una sección hexagonal típicamente barroca. De arriba abajo, describe primero la boca de la lámpara, en forma de "boselón" (bocel), formada por "seis lienzos que baxan forrando el herraje de dicha

${ }^{6}$ SANZ SERRANO, Ma Jesús: La orfebrería sevillana..., op. cit., pp. 196-200

${ }^{7}$ ACS, Fondo Histórico..., leg. 11306, s. f. Ocupa esta parte el folio 1v.

${ }^{8}$ ACS, Ibidem, s. f. (fol. 1v).

${ }^{9}$ ACS, Ibidem, s. f. (fol. 1v).

${ }^{10}$ Estas flores frontales de 4 pétalos, que aparecen en el dibujo desflecados y algo puntiagudos, podrían ser "rosas de cuatro pétalos", muy frecuentes desde 1.700. De hecho, se parecen mucho las flores dibujadas de esta lámpara a las rosas de cuatro pétalos que adornan, grabadas aquí, el borde de una lámpara conservada en la Iglesia de San Julián. Véase SANZ SERRANO, Ma Jesús: La orfebrería sevillana..., op. cit., pp. 197, 233 y 383 (fig. 98).

${ }^{11}$ ACS, Fondo Histórico...., leg. 11306, s. f. Ocupa esta descripción los folios 2r-3v. (las cuatro últimas páginas). 
boca" y coronada por una crestería de acantos formada por doce piezas sueltas y atornilladas. Los seis lados de la boca llevan adorno atornillado de hojas de acanto, en los ángulos y en los frentes. En los ángulos aparecen seis enganches volados para las cadenas que adquieren de nuevo la forma de acantos ondulados muy carnosos. Se decoran con cabezas de serafín y florecillas atornilladas tanto "basiadas" como "de chapa" en su lado externo, elementos que no se reflejan en el dibujo, y con flores de mayor tamaño, de 4 pétalos y frontales, en sus laterales, que si aparecen dibujados ${ }^{12}$.

En la media caña inferior, hexagonal, de nuevo vemos la decoración de florecillas atornilladas en los ángulos y los frentes, unas lisas y otras cinceladas. Apreciamos en el dibujo las flores de 4 pétalos recortados que ya vimos en la cabeza de la lámpara.

Bajo el cuerpo anterior se sitúa el vaso principal, de perfil hexagonal y de formas abombadas o bulbosas. Estas formas son claramente barrocas, pero alcanzan aquí un volumen muy acusado que es raro ver en las piezas coetáneas conservadas $^{13}$. El "esqueleto del herraje" queda forrado por "seis lienzos", que de nuevo se adornan con sobrepuestos. En los ángulos, se dice y se aprecia, aparecen hojas de acanto en el arranque y en el remate, quedando en el centro de cada ángulo una florecilla de 4 pétalos, todo atornillado. En el centro de los frentes también aparecen acantos arriba y abajo que pisan sobre guirnaldas de frutas (o "fruteros") que adornan cada uno de los lados. Todos estos adornos van atornillados. No se reproducen en el dibujo los seis escudos de armas que iban atornillados en la parte inferior de los frentes. Tres pertenecían al "Sr. Cardenal" y los otros tres tenían por blasón "la torre y jarras de la Iglesia", lo que parece indicar que la pieza fue ejecutada en Sevilla ${ }^{14}$.

Bajo el cuerpo anterior se desarrolla el remate, formado por media caña decorada con el mismo modelo ya visto de flores de 4 pétalos sobrepuestas, un elemento acampanado invertido con gallones y gran hoja de acanto como base y varios toros y medias cañas alternados como culminación (su decoración ni se explica ni se aprecia con claridad en el dibujo).

A continuación vuelven a describirse, con otros detalles, las "cartelas" o enganches de las cadenas situados en la boca de la lámpara, y el vaso destinado a cobijar la luz. De este último destaca el uso de pequeñas y rígidas "cadenas"

${ }^{12}$ ACS, Ibidem, s. f. (fols. 2r y 2v).

${ }^{13}$ A la misma tipología podrían adscribirse algunas lámparas de la Capilla Real de la Catedral de Sevilla y de la Iglesia de los Venerables. Son hexagonales, a veces con enganches en forma de acantos, pero mantienen la forma de plato, menos plano que en el Renacimiento, sin llegar al desarrollo volumétrico y de altura que muestra la lámpara del documento. Véase para esas lámparas SANZ SERRANO, M ${ }^{\mathrm{a}}$ Jesús: La orfebrería sevillana..., op. cit., pp. 233 y 383 (fig. 97).

${ }^{14}$ ACS, Fondo Histórico..., leg. 11306, s. f. (fol. 2v). 
("rabisas") de hierro que lo sujetan a la boca de la lámpara, formadas y decoradas por sigmas ("ojas") y "encomiendas de San Juan"15. Este elemento rígido de sujeción ("rabisa" o "rabiza") es algo distinto al que se conserva en la lámpara situada hoy en el altar mayor del Sagrario catedralicio, documentada como obra de Juan Laureano de Pina y fechada en 1711, pues en este caso la "rabisa" adquiere la forma de una sola sigma o tallo en " $\mathrm{S}$ "16.

Finalmente se indica que en el interior del bocel que formaba la boca de la lámpara iban atornilladas y cogidas "por la banda de adentro" unas cartelas ("de fierro forradas en plata") con el nombre del patrono y del autor de la obra ${ }^{17}$.

Se remata el documento señalando que sin faltar pieza alguna se entregó al Sr. Don Juan Bautista Cabaleri, quien mandó se colgase en su sitio. De estas palabras se deduce que fue desmontada, tal vez para su limpieza o corroboración del estado, y vuelta a colgar en un lugar que no conocemos del Sagrario. Sobre D. Juan Bautista Cabaleri, suponemos que se refiere al canónigo Don Juan Cavalieri y Funes, encargado de diversas obras catedralicias durante la primera mitad del s. XVIII, como la nueva Custodia de oro ${ }^{18}$. Hay un Juan Bautista Cavaleri en la misma época, veinticuatro sevillano, que, pese a la más exacta coincidencia de su nombre con el mencionado en el documento, entendemos no tiene relación con este último ${ }^{19}$.

El documento finaliza con una tabla donde se recoge el peso de las distintas piezas y el peso final, deduciéndose de ello que era una lámpara de grandes dimensiones.

Escasos son los datos y conclusiones que se pueden extraer de un documento muy funcional aunque muy detallado en lo descriptivo. Sin duda, su gran valor está en los dibujos que, junto con el texto, permiten hacernos una idea muy exacta de la estructura, técnica y ornamento de esta excepcional lámpara de plata que no hemos localizado.

${ }^{15}$ ACS, Ibidem, s. f., (fol 3r).

${ }^{16}$ Véase DONADO CAMPOS, Inmaculada: "Una nueva obra del platero Juan Laureano de Pina : la lámpara del Sagrario de Sevilla", Archivo Hispalense, t. LXXI, n 216, 1988, pp. 189-192. Nada tiene que ver esta lámpara con la dibujada en el documento que analizamos, aunque deben ser coetáneas.

${ }^{17}$ ACS, Fondo Histórico..., leg 11306, s. f. (fols 3r y 3v).

${ }^{18}$ SANZ SERRANO, María Jesús: "La Custodia de oro de la Catedral de Sevilla", en Estudios de Platería. San Eloy 2003, Murcia, 2003, pp. $569-594$ (p. 575).

${ }^{19}$ Además de veinticuatro, fue Hermano Mayor de la Santa Caridad. En dos ocasiones se documenta su existencia en torno a los años 20 del s. XVIII. Véanse MONTERO DE ESPINOSA, José María: Antigüedades del Convento Casa Grande de San Agustín de Sevilla y noticias del Santo Crucifijo que en él se venera, Sevilla, 1817, p. 32; FALCÓN MÁRQUEZ, Teodoro: "Leonardo de Figueroa, artífice de la torre del Hospital de la caridad de Sevilla", Laboratorio de Arte, no 16, 2003, pp. 501-506 (p. 502). 
La estructura reúne los elementos propios del Barroco local (lámpara de "plato" con movimiento fluido de la forma, interés por los perfiles poligonales, gusto por elementos bulbosos y contrapuestos), si bien se aprecia un desarrollo del volumen y la altura más acentuado que el visto en otras piezas sevillanas de la época.

En la minuciosa descripción técnica, destacan el uso de almas de hierro en los elementos más grandes para el posterior recubrimiento de plata y especialmente los procedimientos decorativos. En efecto, la mayor parte del ornato es sobrepuesto y en bastantes casos el adorno es también funcional, por el uso frecuente de tornillos en forma de flor. Esta decoración sobrepuesta, atornillada o soldada (en forma de chapa o de pieza cincelada o fundida) es muy diferente a la decoración preferentemente cincelada, repujada o grabada que domina el período. Los temas son los habituales del momento, aunque en una gama reducida: flores frontales de 4 o 5 pétalos, acantos carnosos y algunas cabezas de serafines y cogollos de frutos.

El peso recogido al final del documento revela que se trataba de una pieza grande, pues sus 423 marcos de plata (unos $97 \mathrm{kgs}$, no se incluye el peso de las piezas de hierro), duplican, por ejemplo, los 190 marcos que por contrato pesó la ya citada lámpara realizada por Juan Laureano de Pina para el Sagrario ${ }^{20}$. Por desgracia, no se especifican medidas de ningún tipo.

Tampoco se incluyen ni fechas de ejecución ni los nombres del patrono, del que sólo sabemos que era cardenal, y el autor, lo cual limita mucho el alcance de nuestro análisis.

No hemos localizado la pieza en la Iglesia del Sagrario, donde ya en los años 70 del s. XX parece que no se hallaba, pues así lo demuestra el inventario de Sancho Corbacho ${ }^{21}$. Tampoco la hemos visto en la Catedral de Sevilla ni en los catálogos publicados de su Tesoro.

Fecha de recepción: 18 de septiembre de 2014

Fecha de aceptación: 28 de septiembre de 2014

20 DONADO CAMPOS, Inmaculada: "Una nueva obra del platero Juan Laureano...", op. cit., p.191.

${ }^{21}$ SANCHO CORBACHO, Antonio: El Sagrario de la S. I. Catedral de Sevilla. Sus obras de arte, Sevilla, 1981, pp. 22, 28 y 34-38. Sólo se mencionan 3 lámparas barrocas, dos pequeñas en la Capilla del Rosario y la lámpara de Pina del Presbiterio. 


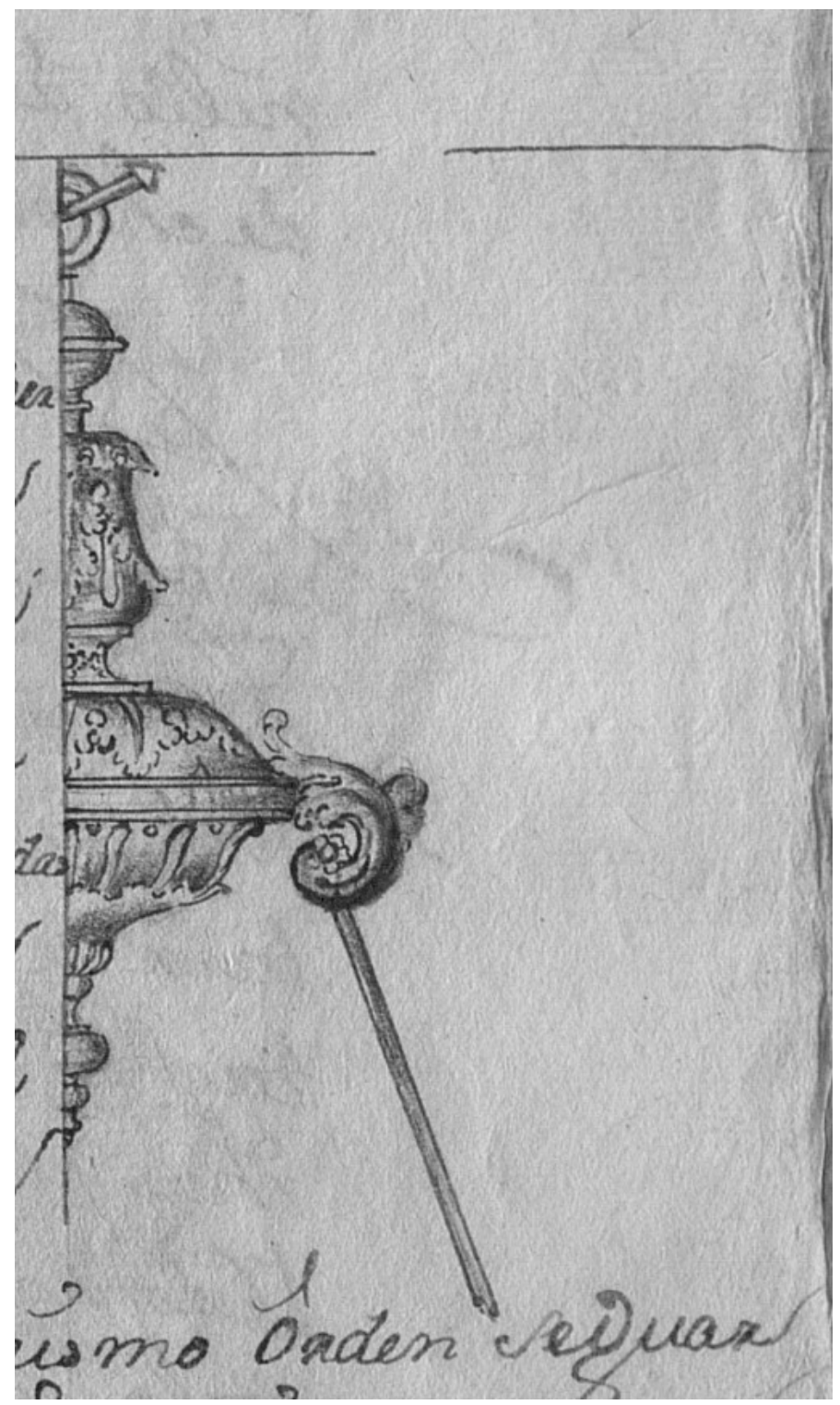

Figura 1. Dibujo de la lámpara desaparecida del Sagrario de la Catedral de Sevilla, manípulo, 1743 (foto: ACS). 




Figura 2. Dibujo de la lámpara desaparecida del Sagrario de la Catedral de Sevilla, cadena, 1743 (foto: ACS)

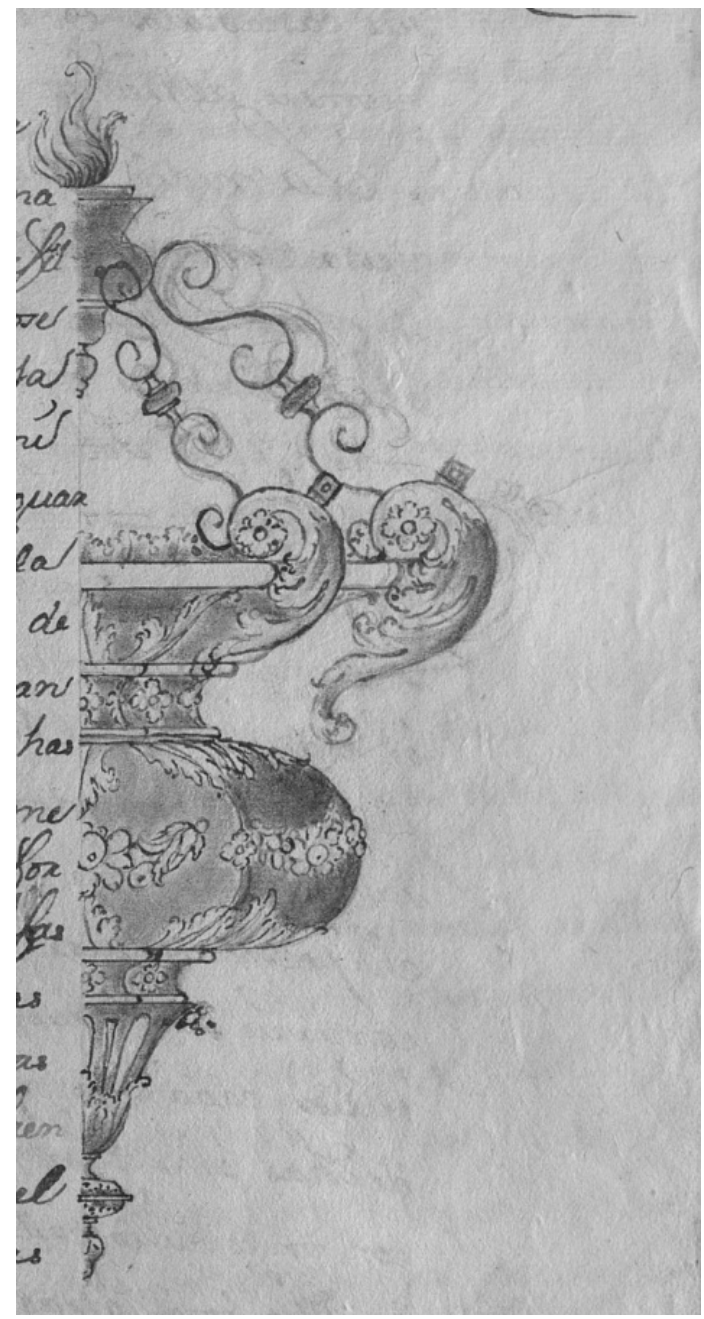

Figura 3. Dibujo de la lámpara desaparecida del Sagrario de la Catedral de Sevilla, vaso o plato principal, 1743 (foto: ACS) 